\title{
Image Processing and Cryo-Transmission Electron Microscopy; Example of Human Proteasome
}

\author{
Hyosun $\mathrm{Choi}^{\dagger}$, Hyunbum Jeon ${ }^{1, \dagger}$, Seulgi Noh, Ohkyung Kwon ${ }^{1}$, Ji Young Mun ${ }^{2, *}$ \\ BK21 Plus Program, Department of Senior Healthcare, Graduate School, Eulji University, Deajeon 34824, Korea \\ ${ }^{1}$ Nanobioimaging Center, National Instrumentation Center for Environmental Management, \\ Seoul National University, Seoul 08826, Korea \\ ${ }^{2}$ Department of Structure and Function of Neural Network, Korea Brain Research Institute, Daegu 41068, Korea
}

\author{
${ }^{\dagger}$ These authors contributed equally to \\ this work. \\ *Correspondence to: \\ Mun JY, \\ (iD) http://orcid.org/0000-0003-0820-6233 \\ Tel: $+82-53-980-8470$ \\ Fax: +82-53-980-8359 \\ E-mail: mjy1026@gmail.com
}

Received March 12, 2018

Revised March 27, 2018

Accepted March 27, 2018
Cryo-transmission electron microscopy (cryo-TEM) allows us to perform structural analysis of a analyses of large protein complexes, which are difficult to analyze using X-ray crystallography or nuclear magnetic resonance. The most common examples of proteins used are ribosomes and proteasomes. In this paper, we briefly describe the advantage of cryo-TEM and the process of two-dimensional classification by considering a human proteasome as an example.

Key Words: Cryo-transmission electron microscopy, Proteasome, Two-dimensional classification, Negative staining

\section{INTRODUCTION}

In 1986, Ernst Ruska won the Nobel Prize in Physics for the development of the first electron microscope (EM), a highresolution imaging device that uses an electron beam with a wavelength shorter than that of visible light. After 31 years, in 2017, Jacques Dubochet, Joachim Frank, and Richard Henderson won the Nobel Prize in Chemistry for developing cryo-transmission electron microscopy (cryo-TEM) techniques. They were able to observe the native structure of molecules in vitrified ice by rapid freezing without dehydration instead of drying molecules after the heavy metal stain (the so-called negative staining). In other words, the cooling of water and the immobilization of the molecules allow us to maintain the structure, as the vitrified water will not evaporate under the vacuum of the EM (Dubochet, 2012; Dubochet \& McDowall, 1981; Dubochet et al., 1988). With the cryo-TEM imaging of the vitrified state, Frank and Henderson's group developed a technique in which the threedimensional (3D) reconstruction of complexes is obtained from sets of two-dimensional (2D) projection images. This approach allow us to obtain a higher resolution (Frank, 1975; Frank et al., 1981; Henderson \& Unwin, 1975). The 3D electron microscopy (3DEM) method, which includes image alignment, averaging, and classification, has recently become a next-generation technology in the field of structural biology, as it allows us to identify the structure of protein complexes at the atomic level (Cheng et al., 2015; Merk et al., 2016; Nogales \& Scheres, 2015). In this paper, we describe the overall process, including the negative-staining technique, cryo-TEM, and image processing (not including the 3D reconstruction), using the proteasome as an example.

Proteasome is a key protein involved in one of the two intrinsic pathways to degrade proteins in cells; it can rapidly degrade misfolded proteins by transferring them to the proteasome complex with ubiquitination. Another pathway is the autophagy-lysosome system, which leads to the degradation of the protein through fusion with the lysosomes inside cell.

The human 26S proteasome (Luan et al., 2016), which

(a) This is an open-access article distributed under the terms of the Creative Commons Attribution Non-Commercial License (http://creativecommons.org/licenses/by-nc/4.0) which permits unrestricted noncommercial use, distribution, and reproduction in any medium, provided the original work is properly cited.

Copyrights @ 2018 by Korean Society of Microscopy 
selectively hydrolyzes proteins is a protein complex with a huge protease assembled from at least 32 different standard subunits. It controls various cellular activities by catalyzing biological reactions in a rapid and unidirectional way. Therefore, if the structure of the $26 \mathrm{~S}$ proteasome is modified, its function will change, and a disease will be triggered. In addition, when the mechanism between structure and function is revealed, the information can be used for structure-based drug discovery. Over the past century, researches involved in proteasome studies have provided profound insights into its structure and function, thus greatly contributing to our understanding of cellular processes. However, there are still many open questions on structural changes.

\section{MATERIALS AND METHODS}

\section{Negative Staining}

Protein sample solution was loaded on glow-discharged carbon-coated copper grids. After loading of $5 \mu \mathrm{L}$ protein $(1 \mu \mathrm{g} / \mu \mathrm{L})$ for 1 minute, the grids were stained in $1 \%(\mathrm{w} / \mathrm{v})$ solution of uranyl acetate for 1 minute. The results were imaged using H-7600 TEM (Hitachi, Japan) at $80 \mathrm{kV}$.

\section{Cryo-TEM}

For the cryo-TEM process, $5 \mu \mathrm{L}$ of protein sample solutions ( 1 $\mu \mathrm{g} / \mu \mathrm{L}$ ) were loaded on glow-discharged C-flat holey carbon grids (EMS, USA) and frozen using Vitrobot (Thermo Fisher Scientific, USA) in liquid ethane. The samples were analyzed using Talos L120C TEM (Thermo Fisher Scientific) at the Nanobio imaging center of Seoul National University.

\section{Image Processing}

The EMAN2.1 image processing program was used for boxing particles, contrast transfer function (CTF) correction, particles selection, and reference free class averaging.

\section{RESULTS AND DISCUSSION}

\section{Comparison between Negative Staining and Cryo-TEM}

For the whole duration of the TEM analysis, the EM must be kept in high-vacuum conditions; for the reason, observation
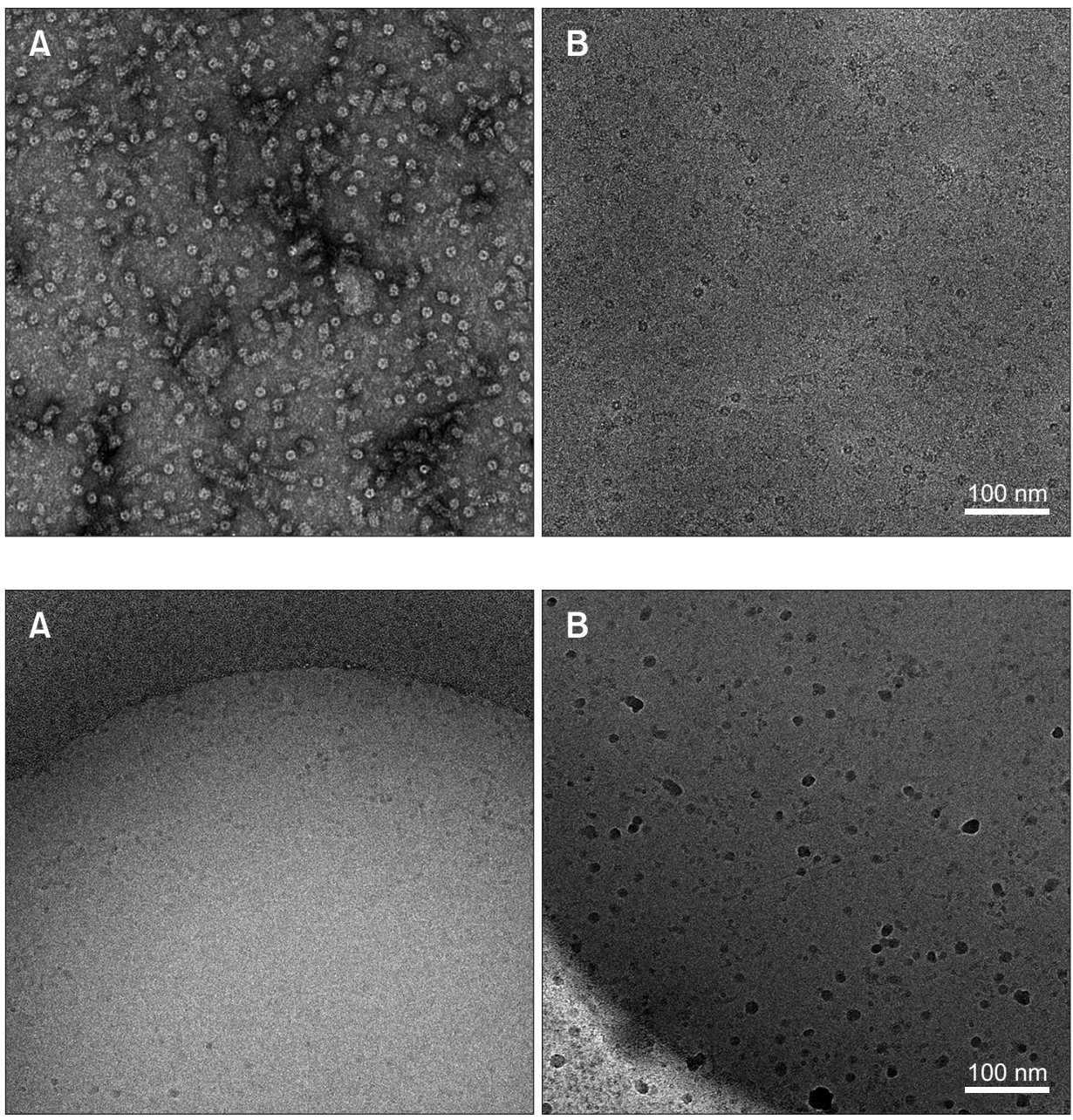

Fig. 1. Comparison between negative staining and cryo-transmission electron microscopy (cryo-TEM). (A) Negative stained proteasomes. (B) Cryo-TEM of proteasomes. Cryo-TEM shows more structural details, compared to negative stained proteins.

Fig. 2. Difficulty of structural study in cryo-transmission electron microscopy. (A) Ununiformed ice. (B) Ethane contamination from plunge freezing. 
of the biological samples presents some limitations. The samples were taken in a dry environment because the level of contrast in the biological sample can be improved using the negative-staining method with metal stains. Several staining solutions, including uranyl acetate and uranyl formate and staining techniques such as the carbon sandwich method, have been adopted to compensate for the structural distortions caused by sample drying (Ohi et al., 2004; Rames et al., 2014). In addition, a random conical tilt method for 3D reconstruction has been applied to perform a detailed structural analysis (Booth et al., 2011; Radermacher et al., 1987). However, since the structure of the protein surrounded by the stain molecules is observed, the resolution is limited (Fig. 1). First of all, negative staining should be tried to check the overall structure and the concentration before using the cryo-TEM method. In addition, in order to keep the structure hydrated with an improved signal-to-noise ratio (SNR), cryonegative staining can be applied (De Carlo \& Stark, 2010).

\section{Cryo-TEM}

Ice vitrification for cryo-TEM is the phenomenon in which water changes into an amorphous vitreous ice state when it is rapidly cooled down to a cryogenic temperature (liquid nitrogen temperature, i.e., approximately $-190^{\circ} \mathrm{C}$ ) (Dubochet et al., 1988). Biological samples preserved in vitrified ice are kept at a very low temperature inside the cryo-TEM and they can be observed without dehydration. Therefore, there is no structural distortion caused by drying. In addition, the resolution limit, which is due to the electron scattering of the staining solution surrounding the sample in negative staining, can be avoided. The ice must be vitrified; however, thick ice or contaminants make the structural analysis difficult (Fig. 2 ). Even though we have enough thin vitrified ice, radiation damages caused by the electron beam can alter the protein structure. In order to minimize such structural alterations (Baker \& Rubinstein, 2010), it is necessary to get the imaging in low-dose conditions. In other words, the area to be imaged must not to be exposed until the image is actually taken for high-resolution imaging because the accumulation of high energy electrons can break molecular bonds and destroy the sample. All image calibration and focusing are done in a nearby area. Moreover, the recent groundbreaking results in terms of high-resolution imaging were obtained from the use of direct detectors and recording of movies (Brilot et al., 2012; Campbell et al., 2012).

\section{Single Particle Analysis (Class Averaging)}

Another limitation in achieving high resolution with cryoTEM is the low contrast of sample; thus, averaging identical particles can be used to improve the resolution. A number of software packages such as SPIDER (Frank et al., 1981), EMAN2 (Tang et al., 2007), FREALIGN (Grigorieff, 2007), RELION (Scheres, 2012), and SPARX (Hohn et al., 2007) have been developed and are still being improved. The low-
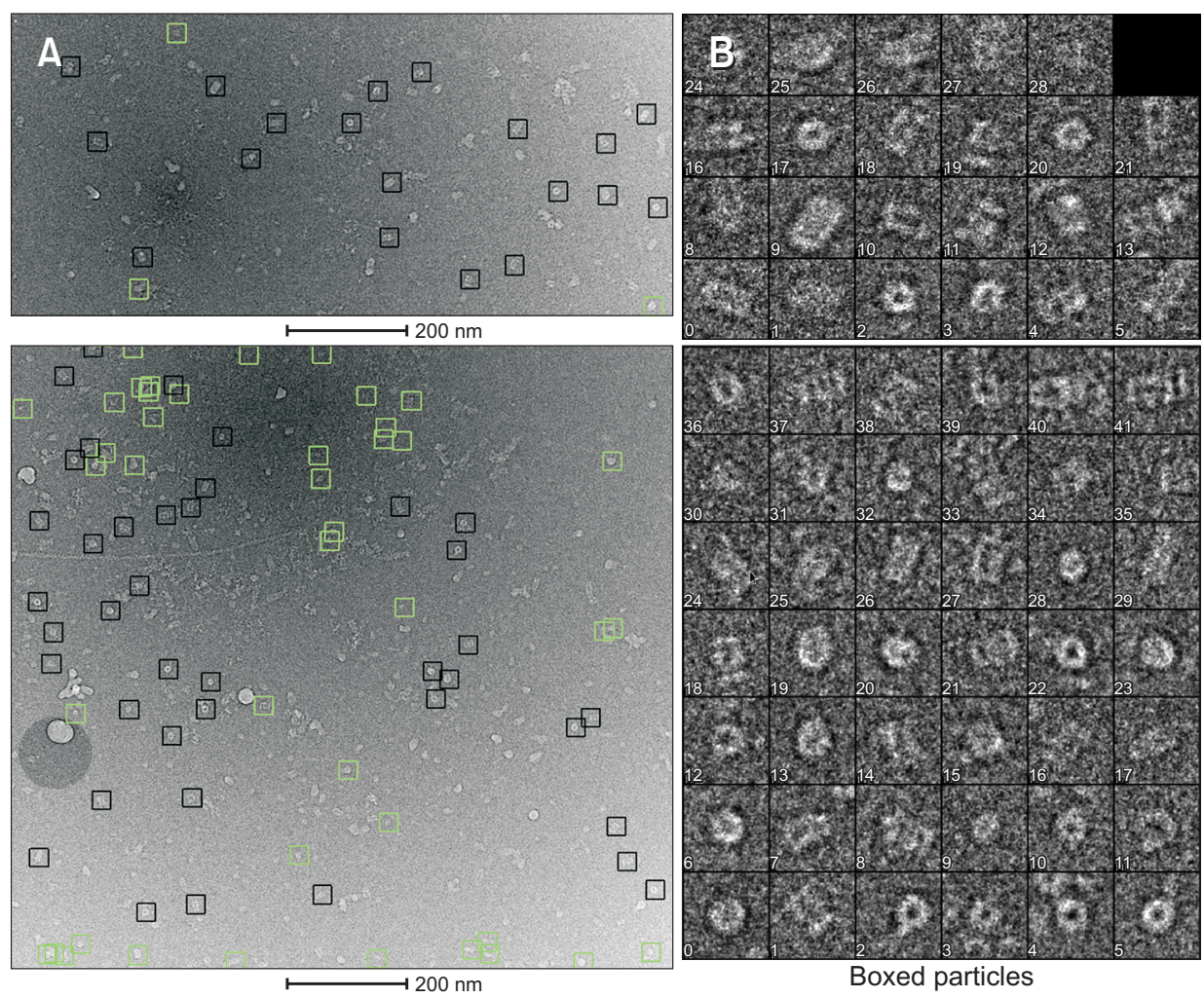

Fig. 3. Semi-automated boxed particles of proteasome in EMAN software. The e2boxer was used for interactive boxing. (A) Semi-automated boxed particles in images. (B) Boxed particles. 


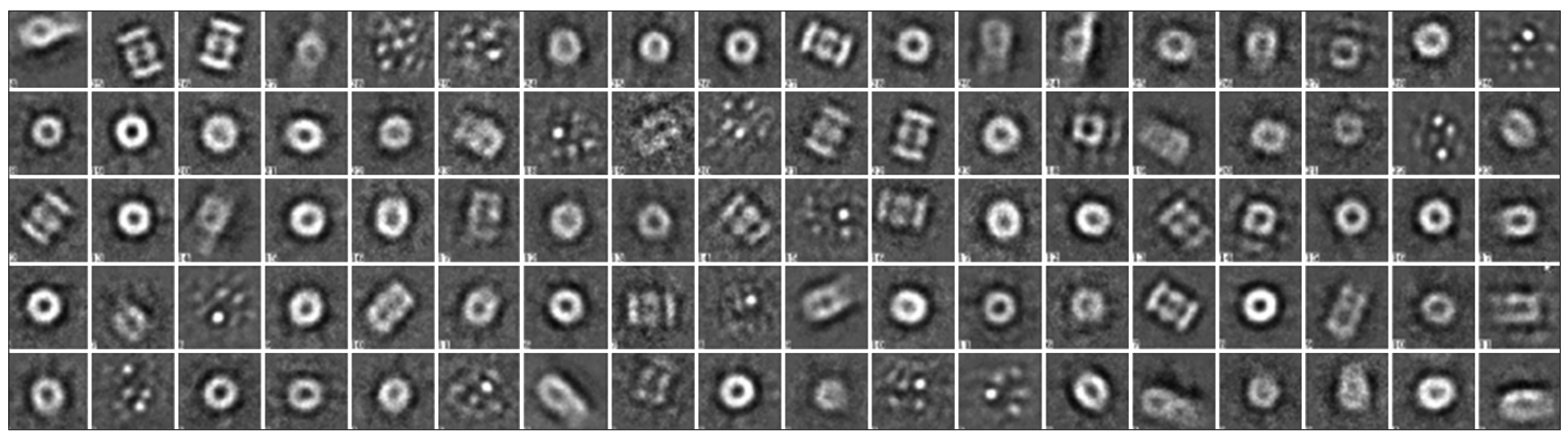

Fig. 4. Class averaging before the elimination of bad particles. These images show the $20 \mathrm{~S}$ proteasome at top view and side view.

contrast cryo-EM boxed images are aligned to same direction, and averaging of large number of particles is done to improve the SNR. Fig. 3 and 4 show the image averaging procedure. The image processing called class averaging of single particle analysis (SPA) acquires a large number of images of the same protein particles; then, in-plane rotation and translation of each image are performed to evaluate the similarities based on cross correlations, and divided for the number of groups through averaging. It is an essential part of image analysis to identify homogeneity and particle orientations. To perform such identifications, the particles are first semi-automated boxed (Fig. 3), and then, the data quality is calculated using CTF parameters, including spectral SNR. In order to compare images of protein particles located in different directions, averaged images generated by randomly dividing data are compared and analyzed through several iterations. The relative rotation angle and defocus value are stored together. The averaged images can be used as an initial model through the evaluation of good/bad particles and later used for $3 \mathrm{D}$ reconstruction.

\section{CONCLUSIONS}

The structural study of protein using cryo-TEM in high resolution is a breakthrough approach that can overcome the limits of X-ray crystallography. The fitting of the domain structure obtained by X-ray or nuclear magnetic resonance to the complex structure obtained by $3 \mathrm{D}$ reconstruction through cryo-TEM and image processing allows us to analyze the interactions between domains or individual proteins. In addition, beyond the structural analysis of purified proteins, analytical methods have been developed to identify intact protein structures in cells. For example, a section of vitrified cells is analyzed by cryo-EM tomography, and a subtomogram averaging can be used for the structural analysis of proteins. Cryo-TEM and 3DEM fields are expected to reach the peak of their development, especially after the Nobel Prize in Chemistry 2017.

\section{CONFLICT OF INTEREST}

No potential conflict of interest relevant to this article was reported.

\section{ACKNOWLEDGMENTS}

We thank Min Jae Lee of the Seoul National University College of Medicine for the gift of proteins. This work was supported by the Basic Science Research Program through the National Research Foundation of Korea (NRF) funded by the Ministry of Science, ICT \& Future Planning (2015R1C1A1A02037153).

\section{REFERENCES}

Baker L A and Rubinstein J L (2010) Radiation damage in electron cryomicroscopy. Methods Enzymol. 481, 371-388.

Booth D S, Avila-Sakar A, and Cheng Y (2011) Visualizing proteins and macromolecular complexes by negative stain EM: from grid preparation to image acquisition. J. Vis. Exp. (58). doi: 10.3791/3227.

Brilot A F, Chen J Z, Cheng A, Pan J, Harrison S C, Potter C S, Carragher B, Henderson R, and Grigorieff N (2012) Beam-induced motion of vitrified specimen on holey carbon film. J. Struct. Biol. 177, 630-637.

Campbell M G, Cheng A, Brilot A F, Moeller A, Lyumkis D, Veesler D, Pan J, Harrison S C, Potter C S, Carragher B, and Grigorieff N (2012) Movies of ice-embedded particles enhance resolution in electron cryomicroscopy. Structure 20, 1823-1828.

Cheng Y, Grigorieff N, Penczek P A, and Walz T (2015) A primer to singleparticle cryo-electron microscopy. Cell 161, 438-449. 
De Carlo S and Stark H (2010) Cryonegative staining of macromolecular assemblies. Methods Enzymol. 481, 127-145.

Dubochet J (2012) Cryo-EM-the first thirty years. J. Microsc. 245, 221224.

Dubochet J, Adrian M, Chang J J, Homo J C, Lepault J, Mcdowall A W, and Schultz P (1988) Cryo-electron microscopy of vitrified specimens. Q. Rev. Biophys. 21, 129-228.

Dubochet J and Mcdowall A W (1981) Vitrification of pure water for electron microscopy. J. Microsc. 124, 3-4.

Frank J (1975) Averaging of low exposure electron micrographs of nonperiodic objects. Ultramicroscopy 1, 159-162.

Frank J, Verschoor A, and Boublik M (1981) Computer averaging of electron micrographs of $40 S$ ribosomal subunits. Science $\mathbf{2 1 4}$, 1353-1355.

Grigorieff N (2007) FREALIGN: high-resolution refinement of single particle structures. J. Struct. Biol. 157, 117-125.

Henderson R and Unwin P N (1975) Three-dimensional model of purple membrane obtained by electron microscopy. Nature 257, 28-32.

Hohn M, Tang G, Goodyear G, Baldwin P R, Huang Z, Penczek P A, Yang C, Glaeser R M, Adams P D, and Ludtke S J (2007) SPARX, a new environment for Cryo-EM image processing. J. Struct. Biol. 157, 4755.

Luan B, Huang X, Wu J, Mei Z, Wang Y, Xue X, Yan C, Wang J, Finley D J,
Shi Y, and Wang F (2016) Structure of an endogenous yeast 265 proteasome reveals two major conformational states. Proc. Natl. Acad. Sci. U S A 113, 2642-2647.

Merk A, Bartesaghi A, Banerjee S, Falconieri V, Rao P, Davis M I, Pragani R, Boxer M B, Earl L A, Milne J L S, and Subramaniam S (2016) Breaking cryo-EM resolution barriers to facilitate drug discovery. Cell 165, 1698-1707.

Nogales E and Scheres S H (2015) Cryo-EM: a unique tool for the visualization of macromolecular complexity. Mol. Cell 58, 677-689.

Ohi M, Li Y, Cheng Y, and Walz T (2004) Negative staining and image classification--powerful tools in modern electron microscopy. Biol. Proced. Online 6, 23-34.

Radermacher M, Wagenknecht T, Verschoor A, and Frank J (1987) Threedimensional reconstruction from a single-exposure, random conical tilt series applied to the $50 \mathrm{~S}$ ribosomal subunit of Escherichia coli. J. Microsc. 146, 113-136.

Rames M, Yu Y, and Ren G (2014) Optimized negative staining: a highthroughput protocol for examining small and asymmetric protein structure by electron microscopy. J. Vis. Exp. (90), e51087.

Scheres S H (2012) RELION: implementation of a Bayesian approach to cryo-EM structure determination. J. Struct. Biol. 180, 519-530.

Tang G, Peng L, Baldwin P R, Mann D S, Jiang W, Rees I, and Ludtke S $J$ (2007) EMAN2: an extensible image processing suite for electron microscopy. J. Struct. Biol. 157, 38-46. 